\title{
Aiding the operator's anticipatory behaviour: The design of process state information
}

\author{
G.P.J. Spenkelink \\ Ergonomics Group, Twente University, Enschede, Netherlands
}

\begin{abstract}
Video display units have penetrated into modern control rooms, replacing conventional instrumentation as a means of information presentation to the operators. In four experiments simulating a small chemical plant process, several process variable and presentation characteristics were investigated. The experiments focused on the early detection of 'oncoming off-normals' (ocons) - deviations in the process that have just begun to appear. The results show that the way information is presented and some characteristics of the signals presented affect detection performance. It is also suggested that the presence of historical information, in the form of trends, seriously hampers the early detection of oncoming off-normals.
\end{abstract}

\section{Keywords: Human performance, information, visual display units, process plant, detection, discrimination tasks}

\section{Introduction}

In large-scale process industries, operators in centralised control rooms are a vital link in the production process. The operators are directly responsible for the smooth operation of the plant. As field research has shown, operators continuously assess the process state (see, for example, Dallimonti (1980), van Droffelaar (1975) and Zwaga and Veldkamp (1984)). This assessment appears to be a very important aspect of operators' conception of their job.

Observations that operators do not wait for alarm signals, but anticipate off-normal events (Zwaga and Veldkamp, 1984) and act accordingly, may be regarded as evidence for the continuous nature of the assessment task.

In order to be able to evaluate the state of the process and anticipate deviations from normal operation, the operator needs information about the process. Not only the content of the information (which of the process variables are being shown), but also the way in which the information is presented (how the variables are presented) may hamper or facilitate operator behaviour. Design of the information should meet the needs of the anticipation tasks. Since these tasks involve discrimination of normal fluctuations of process variables from abnormalities, the operator will be aided by information presentation designed to facilitate these specific discrimination tasks.

In terms of skill, rule and knowledge-based behaviours, knowledge of the process and the application of rules are inevitably present in the anticipation task. However, the actual detection of abnormalities is mainly guided by skillbased behaviour.

Video Display Units (VDUs) have penetrated into the modern control rooms as the main means of information presentation and, to an increasing extent, replace conventional instrumentation. VDUs offer great flexibility with respect to the design of information and therefore provide a good opportunity to adapt the visual interface to the needs of the operator and to the demands of the task.

The laboratory study presented here explores several aspects of the relationship between information design and the anticipatory detection of oncoming abnormal states in an ongoing process.

\section{The experiments}

Effects of six factors on operator performance were studied in four experiments, all under the same simulation of an ongoing process. The main variable of interest, presentation mode, was varied between blocks within each experiment. Four factors of signal characteristic, deviation frequency, deviation amplitude and deviation angle were each varied between blocks in the filtering, frequency, amplitude and angle experiments respectively, and held fixed for the other three experiments (signal characteristic was, in fact, randomly varied within blocks for all experiments except the filtering one). For trends only, whether these were combined or separated was varied non-systematically within blocks. Table 1 provides an overview of the experiments and the associated experimental conditions; more information on the independent variables is provided below. Each experiment had 2 (mode) $\times 3$ (levels of variable of interest) blocks.

Below we describe in some detail the process and the experimental set-up common to all four experiments. Then the individual experiments are described.

\section{The process}

The simulated process was representative of a small petroleum refinery. This simulation has been developed 
Table 1: Overview of experiments, showing levels of experimental and fixed variables. Refer to the text for an explanation of the independent variables and the meaning of the levels

\begin{tabular}{|c|c|c|c|}
\hline Experiment & Variable & Varied & Factor level(s) \\
\hline \multirow[t]{8}{*}{ Filtering } & Presentation mode & $\begin{array}{r}\text { B (etween } \\
\text { blocks) }\end{array}$ & $\begin{array}{r}\text { trend } \\
\text { bar }\end{array}$ \\
\hline & Signal characteristic & $\mathrm{B}$ & 0.98 \\
\hline & & & 0.96 \\
\hline & & & 0.94 \\
\hline & Deviation frequency & $F($ ixed $)$ & 15 \\
\hline & Deviation amplitude & $\mathrm{F}$ & 30 \\
\hline & Deviation angle & $\mathrm{F}$ & $24 \cdot 5$ \\
\hline & Separation of trends & $\begin{array}{l}\text { W(ithin } \\
\text { blocks) }\end{array}$ & $\begin{array}{l}\text { combined } \\
\text { separated }\end{array}$ \\
\hline
\end{tabular}

Frequency Presentation mode B trend

0.98

0.96

0.94

Deviation frequency $B$

Deviation amplitude $F$

Deviation angle $F$

Separation of trends $W$

\begin{tabular}{|c|c|c|c|}
\hline \multirow[t]{10}{*}{ Amplitude } & Presentation mode & B & trend \\
\hline & Signal characteristic & $w$ & 0.98 \\
\hline & & & 0.96 \\
\hline & & & 0.94 \\
\hline & Deviation frequency & $F$ & 15 \\
\hline & Deviation amplitude & B & 40 \\
\hline & & & $\begin{array}{l}30 \\
20\end{array}$ \\
\hline & Deviation angle & $F$ & $30 \cdot 6$ \\
\hline & Separation of trends & $w$ & combined \\
\hline & & & separated \\
\hline \multirow[t]{10}{*}{ Angle } & Presentation mode & $\mathrm{B}$ & trend \\
\hline & Sianal characteristic & w & 0.98 \\
\hline & & & 0.96 \\
\hline & & & 0.94 \\
\hline & Deviation frequency & $F$ & 15 \\
\hline & Deviation amplitude & $\mathrm{F}$ & 20 \\
\hline & Deviation angle & B & $24 \cdot 5$ \\
\hline & & & $30 \cdot 6$ \\
\hline & & & $34 \cdot 3$ \\
\hline & Separation of trends & $w$ & $\begin{array}{l}\text { combined } \\
\text { separated }\end{array}$ \\
\hline
\end{tabular}

over several years in close co-operation with experts in the field of process control and instrumentation systems. Process operation experts from three process industries have given special attention to the degree of reality of the structure of the simulated process and of the signals presented.

Three types of process units were distinguished for the simulation: furnaces $(F)$, distillation columns $(C)$ and chemical reactors $(R)$. There are 29 such units, each providing information about a number of variables. They are split into four groups. Although in real processes inter-dependencies exist between many process variables, all our simulated variables behaved independently. The chosen level of presentation corresponded to an overview task; information about many process variables was displayed simultaneously.

Disturbances (called oncoming off-normals or 'ocons') in the process were generated at random times in variables that were randomly selected within the constraints of the experimental design. These disturbances were simply constant rate functions, added to the signal. Alarms did not appear in this simulation and all disturbances were regarded as indicative of an off-normal situation.

The process could be represented in two modes: as trends (Fig. 1, page 213), showing present and past values, and as bargraphs (Fig. 2, page. 213), showing only present values. Both figures show one of four different displays, each of which is called a 'group' of the units. Thus group 2, which is shown, has seven units: furnaces F4, F5, F6, reactors R2 and $\mathrm{R} 3$, and distillation columns $\mathrm{C} 2$ and $\mathrm{C} 3$.

The underlying process variables all fluctuated in a controlled manner around a baseline value, the setpoint. Deviations were either increasingly (by adding a constant rate function) or decreasingly (by subtracting the function) directed.

\section{Apparatus}

The process simulation was accomplished with a configuration consisting of two computers (an 'LSI' and a 'micro-PDP') and a graphical system (Ramtek 9460) coupled to a high-resolution colour CRT. The LSI controlled the experiments, while the PDP controlled the graphical system. The picture resolution used on the CRT was $640 * 512$ display elements. The size of the display was 350 by $255 \mathrm{~mm}$.

Three keyboards were installed in front of the colour display (Fig. 3).

The upper, large, keyboard depicted the process structure and could be used for selection of pictures for display although this facility was not used in the present experiments. Only the keys labelled NXT and CLR were used for selecting the next 'group' for display and clearing the screen, respectively.

The two smaller keyboards were used for the identification of the ocons. These were also used for entering and correcting responses. The right one was used for entering unit type ( $\mathrm{C}, \mathrm{R}$ or $\mathrm{F})$, a percentage indicating subjective certainty about the response $(20,40,60,80$ or $100 \%)$ and the enter key for acknowledging the response. The left keyboard was used for entering the unit number ( 1 to 9) and the identification of the variable within a unit

The keys of the numerical keypad were not only numbered, but also coloured (in rows) corresponding to the colours of the trends; the keys 1,3 and 6 were blue, 2, 4 and 7 were brown, 5 and 8 were white and 9 was pink. 

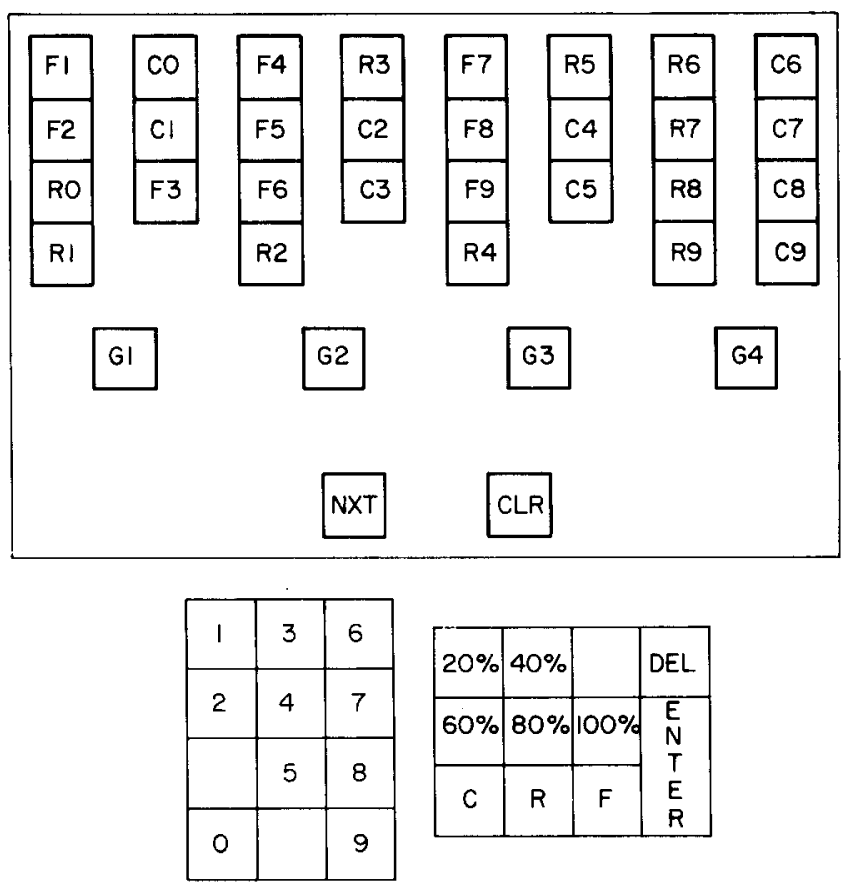

Fig. 3 The keyboards used in the experiments. The upper large keyboard provided an overview of the process structure. Only the keys labelled NXT and CLR were used. The two smaller keyboards below were used for the identification of process units. Keys 1, 3, 6 coloured blue; 2, 4, 7 brown; 5,8 white; and 9 pink

\section{Experimentation room}

Experimentation took place in a specially isolated room in order to avoid disturbing the subjects. The experimenter consoles for starting up and monitoring the experiments were placed outside the experimentation room.

The room was lit with a spotlight situated above and behind the display, projecting its light on the wall behind the display. In this way the contrast between display and direct surroundings was not too high, there was no glare and the illumination level thus provided ensured comfortable use of the keyboards.

\section{Subjects}

All subjects were students in the highest grade of a Polytechnical school and were familiar with the basics of process operation. Participation was paid and voluntary. None of the subjects took part in more than one experiment. Each of the experiments was executed with six subjects.

\section{Task}

The task of the subjects was to detect, identify and report ocons at the earliest possible stage. The information available was split into four quarters, and the subjects had only one quarter of the process information available at a time by pressing a button (the one labelled 'NXT' in Fig. 3) they could ask for the next quarter of information to be displayed. The order in which the four quarters were presented was fixed. Subjects were instructed to keep track of all four groups of information and to report any ocons found as soon as possible. After clearing the screen by pressing the CLR key, an ocon could be reported by entering on the relevant keyboard the unique identification code of the process variable in question. For trends, this consisted of the code of the process unit, the colour of the trend and the record in which it appeared within the unit. The bargraph variable code was made up of the unit code and the number of the variable within the unit. This number corresponded to the position of the bar. Both for trends and bars a variable could be identified by making three keystrokes. For trends, these were: the C, R or F key on the right keyboard, a numerical key from the left keyboard (indicating the unit-number) and one of the numerical keys from the left keyboard representing the correct combination of trend colour and trend record (e g, 'F'-4'-blue key in first column). For bars, the keystrokes were: the $\mathrm{C}, \mathrm{R}$ or $\mathrm{F}$ key, the unit number and the position number of the bar within the record as counted from the left (eg, 'F'-4'-' 1 ').

In addition, subjects were requested to indicate how certain they were of their response being correct, by pressing one of the percentage buttons $(20,40,60,80$ or $100 \%$ sure). The keyed information was echoed in the lower right corner of the display. Until the ENTER key was hit the keyed sequence could be edited. An example of a full response would be: 'F'.4'-blue key in first column (='1')-'80\%'-'ENTER'.

If the reported variable contained an ocon, its state reverted to normal, obeying the same rule that generated the ocon. If an ocon was reported in a variable that did not contain one, the report did not have any effect on the behaviour of the variable.

\section{Procedure}

Some days before taking part in an experiment the subjects received a written instruction, explaining the structure of the process, the layout of the keypads and the information displayed on the screen. The concept of ocons was introduced: ocons were described as deviations in signals as a consequence of disturbances in the process. It was also explained that these deviations would show up as increasing departures from the setpoint. The experimental task was explained in detail, with examples.

An experiment consisted of six experimental blocks, lasting $15 \mathrm{~min}$ each. In each block one condition was presented, meaning that the variable of interest was fixed within and varied between blocks. Three of the six blocks were presented in trend mode, the other three in bargraph mode.

In advance of the experiment, subjects practised the ocon detection task (and were given feedback about their actions) for about 15 min using the presentation mode with which they were to start (trend or bar). They also had the opportunity to ask any questions about the task, the structure of the process or the experimental procedure. Before the first experimental block which contained the other presentation mode, subjects again practised using the new mode for $15 \mathrm{~min}$.

Two subjects participated in the experiment at the same time. Experimental blocks were administered in turn, so that one subject could rest for $15 \mathrm{~min}$ or fill out a questionnaire whilst the other completed an experimental block. With each presentation mode, the blocks were administered in different orders in a balanced way. Half of the subjects started with trends, the other half with bargraphs. 
After completing the three blocks in one of the presentation modes, the subjects filled out a questionnaire.

\section{Independent variables}

In each of the four experiments, each of which contained six blocks, the presentation mode and one of the other independent variables were varied (as shown in Table 1). All remaining variables were kept constant at one of the factor levels.

The independent variables in the experiments were:

- presentation mode (either bargraphs or trends)

- signal characteristic (level of applied filtering)

- deviation frequency (frequency with which ocons occurred)

- deviation amplitude (maximum size of the deviation)

- deviation angle (the rate of increase of the deviation)

- separation of trends (setpoint position relative to other trends)

Below we deal with these independent variables in more detail.

\section{Presentation mode}

The information about the process variables could be presented in two ways: by means of bargraphs and by means of trends (see Figs. 1 and 2).

In previous research (Pousse, 1983; Weerdmeester, 1986) bargraph presentation was found to be superior to trend presentation with respect to the performance of experimental subjects. However, the results reported by Pousse were obtained with still pictures in static simulations. Also, in the research reported by Weerdmeester, presentation mode was confounded with the hierarchical level within the simulated process, with an advantage for bars. In a dynamic simulation, therefore, there is no strong prior evidence in favour of bargraph presentation with respect to ocon detection performance; indeed, experts tended to favour the trend presentation.

When bargraph presentation was used, the actual value of one quarter of the process variables (in seven or eight process units) was given on the display, each bar representing one variable of one process unit. The vertical length of the bar indicated the actual value and was updated every $15 \mathrm{~s}$.

The trend displays showed both actual and historical values of the process variables. Again, one quarter of the process variables was displayed simultaneously. As with bargraphs an update took place every $15 \mathrm{~s}$. Each trend consisted of 60 data points and, the update frequency being $15 \mathrm{~s}$, a history of 15 min was visible. The time axis ran from the left to the right. As can be seen in Fig. 1, the variables of each unit were split into three 'records'. The exact number of trends in each record depended on the particular type of unit: a record consisted of one, two, three or four trends. The buildup time for trend pictures was $10 \mathrm{~s}$, for bargraph pictures $1 \mathrm{~s}$. When the subjects requested a new picture, therefore, they had to wait longer with trends than with bars.

Signal characteristic (varied in the filtering experiment)

For trends, van Schaik (1984) found that signals with less filtering were associated with better ocon detection performance. Weerdmeester (1986), on the contrary, indicates that filtering of trends may improve detection performance. Therefore, in the present research there was no expectation concerning the direction of performance effects of filtering. Six different signals were assigned to the 220 process variables in both presentation modes. These signals differed from each other in the degree of filtering and in the presence or absence of additional random noise. This totals to 3(levels of filtering) $\times 2$ (levels of random noise) $=6$ different signal types.

The degree of filtering determined the amount of highfrequency fluctuations in the signal, a random sampling of values constituting the least filtering. By manipulating a damping factor different filterings could be attained. The general formula was:

$$
S_{\mathrm{t}}+1=a \times S_{\mathrm{t}}+b \times N+N_{\mathrm{a}}
$$

for the signal with noise, where $S_{\mathrm{t}}+1$ is the value to be computed, $S_{\mathrm{t}}$ the momentary value, $N$ a random number in a two-sided amplitude range. ' $a$ ' and ' $b$ ' are weights. $N_{\mathrm{a}}$ is the added random noise; this value was zero for signals not containing random noise.

For the ' $a$ ' weight, values $0.98,0.96$ and 0.94 were chosen; a higher value corresponds with a more heavily filtered signal with a smoother appearance. The ' $b$ ' weights were calculated to keep the signal within the specified amplitude range of 30 pixels with a certainty of $99.5 \%$. The amplitude range of the added noise was set at 10 pixels vertically.

In the three experiments in which filtering was not investigated, all types of signal appeared. These were distributed randomly over the process units.

\section{Deviation frequency (varied in the ocon frequency experiment)}

Due to human characteristics (information processing capacity, attentional factors, motivation, etc) there will be upper and lower limits to the ocon frequency that can be handled. Therefore, the ocon frequency was varied in one experiment. Since very low frequencies would not yield enough performance data for analysis, three conditions were defined in which 15,20 and 25 ocons respectively were introduced in the process. Equality of performance under these conditions was assured. In the other three experiments, 15 ocons were introduced.

Deviation amplitude (varied in the amplitude experiment)

The amplitude of the ocon refers to the deviation value added in case of a maximally deviating signal, and thus refers to the maximum size of the deviations. Three one-sided values were chosen: 20,30 and 40 pixels. As opposed to the filtering and frequency experiments, in the angle experiment the ocon amplitude was fixed at 20 pixels instead of the intended 30 , but this would not have affected the validity of the experiment

Because several counteracting effects of increasing the ocon amplitude may exist (e g, detection threshold effects, masking of ocons by other signals, differences between trends and bars), no specific differences between the amplitude conditions were expected.

Deviation angle (varied in the angle experiment)

The angle of departure of an ocon determined the speed with which the ocon grew larger. Three angles were chosen: $24 \cdot 5^{\circ}, 30 \cdot 6^{\circ}$ and $34 \cdot 3^{\circ}$, equivalent to $0.50,0.65$ and 0.75 
picture elements (pixels) per update. The implication of using different angles is that the maximum size of the ocons, as defined by the deviation amplitude, is reached after different time intervals.

In the ocon frequency and filtering experiment the ocon angle was fixed at $24.5^{\circ}$, and in the amplitude experiment at $30 \cdot 6^{\circ}$.

Although it seems reasonable to assume that ocon angle affects detection performance, we had no specific hypothesis with respect to the direction of such an effect.

\section{Separation of trends}

In a static simulation experiment, a difference in performance has been found between trends that all shared the same setpoint position on the display and trends that were displayed vertically separated (van Schaik, 1984). Detection performance was better with the former. Experts, however, felt that this could have been an experimental artefact, and in the present experiment they expected better performance with vertically separated trends. The difference between the two types of presentation of trends can be seen in Fig. 1 . The trends of process unit C 3 (lower right), for example, are combined (the setpoint values of all trends in a record share ordinate) but those of unit F4 (upper left), for example, are displayed in a separated fashion, the ordinates of the individual trends in a record being separated by 10 display elements.

It should be emphasised that this factor was not varied in a systematic manner in the present experiments. Separated and combined trends were assigned to process units in an arbitrary fashion.

\section{Dependent variables}

The performance of subjects was measured in terms of the number of ocons detected and falsely reported (hits and false alarms respectively). and of the time needed to find ocons (reaction time, RT). Reaction time was defined as the time interval between the onset of an ocon and the clearing of the screen in order to report the ocon. The ratio of hits

$\overline{\text { hits }+ \text { false alarms }}$

(the hit rate) was calculated. This measure expresses detection performance in terms of detection efficiency.

The number of hits and the number of false alarms are measures of absolute detection performance. In practice, the importance attached to these two measures may vary with the cost (production or safety) of missing ocons or of the actions which may be taken after erroneously detected ocons. The hit rate is a measure of relative detection performance in which changes in detection strategy and detection efficiency (as a result of changing circumstances) may come to expression. In systems where the time that is available for corrective actions is limited, the time needed to detect an ocon (RT) may be critical. Through measures of reaction time, threshold effects regarding signal/ocon discriminability may come to expression (e $\mathrm{g}$, they are related to the increasing size of deviations over time).

The total time that a picture, containing the reported ocon, was actually displayed (selection time) was recorded. The time needed for keying the code of the reported variables was recorded separately. Finally, subjects had to indicate how certain they felt that the reported deviation really/was an ocon, and they completed a questionnaire asking for preferences and an evaluation of performance.

\section{Results and discussion}

In the following sections the results of the experiments are presented. Factors that varied between blocks in only one of the four experiments are described first. The next section deals with presentation mode, which was varied between blocks in all four experiments. Since the separation of trends was analysed post hoc, a separate section is devoted to this factor. The last two sections deal with the keying time results and the subjective data respectively.

\section{Signal and ocon characteristics}

The data were analysed by the Friedman two-way analysis of variance by ranks; only the reaction times were analysed by an ANOVA. All analyses were conducted separately for trend presentation and bargraph presentation data. Interaction effects could not be tested for with the non-parametric analysis: they were only established for RT.

Table 2 presents the most relevant performance data of the four experiments.

In the filtering experiment, the number of false alarms differs significantly across signal characteristics (and the number of hits almost significantly) in the bargraph presentation mode. For trends, a significant hit rate effect is present. The hit rate for trends and bars in the condition with the least filtered signal (filtering 0.94 ) is higher than in the condition with medium filtering. This may indicate that subjects adapted their detection behaviour to the changing demands as the signal became rougher. The selection times did not differentiate between the filtering conditions; therefore, the increase in reaction time for bars in the least filtered condition, although not statistically significant, suggests that subjects did not observe the bars longer in this condition, but merely postponed their decision.

The frequency with which ocons were introduced in the process significantly influenced the hit rate associated with trends displays. From the condition with 15 to that with 20 ocons the hit rate improves substantially. Raising the ocon frequency to 25 did not lead to further improvement.

It can be concluded that even the highest ocon frequency, 25 ocons in $15 \mathrm{~min}$, did not adversely affect performance.

The results of the amplitude experiment indicate that ocons are generally detected before they reach the maximum amount of deviation, since no significant effect of ocon amplitude was demonstrated. The ocons with the smallest amplitude were detected just as reliably as those with larger amplitudes.

Increasing the angle under which the deviations departed (angle experiment) improved the detection of ocons with bars, but not with trends (Fig. 4)

The false alarm frequencies are stable over the three angle conditions. Both for bars and trends the RTs for the largest angle are much shorter than for the smaller two angles (Fig. 5).

Although the hit rate figures are non-significant, the results tentatively indicate an advantage of bars over trends when deviations have large angles. 
Table 2: Performance data of the four experiments with indication of significant effects $(0<0.05)$. Significant differences between the levels of filtering, frequency, amplitude and angle conditions (tested with Friedman ANOVA) are indicated by single underlining. Significant effects of presentation mode (tested with Wilcoxon's matched pairs signed rank test) are indicated by double underlines of pairs of totals.

\begin{tabular}{|c|c|c|c|c|c|c|c|c|c|}
\hline \multirow{2}{*}{\multicolumn{2}{|c|}{$\begin{array}{l}\text { Experiment } \\
\text { and value of } \\
\text { independent } \\
\text { variable }\end{array}$}} & \multicolumn{2}{|c|}{$\begin{array}{l}\text { No of } \\
\text { hits }\end{array}$} & \multicolumn{2}{|c|}{$\begin{array}{c}\text { No of } \\
\text { false alarms }\end{array}$} & \multicolumn{2}{|c|}{$\begin{array}{c}\text { Hit rate } \\
(\%)\end{array}$} & \multicolumn{2}{|c|}{$\begin{array}{c}\text { Mean RT } \\
(s)\end{array}$} \\
\hline & & Bar & Trend & Bar & Trend & Bar & Trend & Bar & Trend \\
\hline \multirow[t]{4}{*}{ Filtering } & 0.98 & 63 & 52 & 19 & 26 & 84 & 70 & 373 & 409 \\
\hline & 0.96 & 44 & 32 & 70 & 45 & 44 & 43 & 359 & 421 \\
\hline & 0.94 & 57 & 23 & $\underline{55}$ & 48 & 54 & $\underline{52}$ & 434 & 358 \\
\hline & & $\overline{164}$ & $\overline{107}$ & $\overline{144}$ & $\overline{119}$ & $\overline{61}$ & $\overline{55}$ & $\overline{389}$ & $\overline{396}$ \\
\hline \multirow[t]{4}{*}{ Frequency } & 15 & 37 & 23 & 32 & 32 & 57 & 47 & 434 & 331 \\
\hline & 20 & 54 & 33 & 41 & 21 & 63 & 68 & 371 & 427 \\
\hline & 25 & 69 & 36 & 40 & 26 & 62 & 67 & 437 & 349 \\
\hline & & $\overline{160}$ & $\overline{92}$ & $\overline{113}$ & 79 & $\overline{61}$ & $\overline{61}$ & $\overline{414}$ & $\overline{369}$ \\
\hline \multirow[t]{4}{*}{ Amplitude } & 40 & 69 & 49 & 39 & 55 & 67 & 58 & 342 & 359 \\
\hline & 30 & 68 & 52 & 50 & 57 & 62 & 60 & 322 & 409 \\
\hline & 20 & 67 & 40 & 54 & 48 & 60 & 58 & 358 & 383 \\
\hline & & $\overline{204}$ & $\overline{141}$ & $\overline{143}$ & $\overline{160}$ & $\overline{63}$ & $\overline{59}$ & $\overline{341}$ & $\overline{384}$ \\
\hline \multirow[t]{4}{*}{ Angle } & $24 \cdot 5$ & 45 & 45 & 32 & 33 & 66 & 67 & 443 & 407 \\
\hline & $30 \cdot 6$ & $\underline{65}$ & 44 & 27 & 38 & 75 & 62 & $\underline{429}$ & $\underline{410}$ \\
\hline & $34 \cdot 3$ & $\underline{72}$ & 52 & 30 & 37 & 74 & 68 & $\underline{323}$ & 371 \\
\hline & & $\overline{182}$ & $\overline{141}$ & $\overline{89}$ & $\overline{108}$ & $\overline{72}$ & $\overline{66}$ & $\overline{398}$ & $\overline{396}$ \\
\hline
\end{tabular}

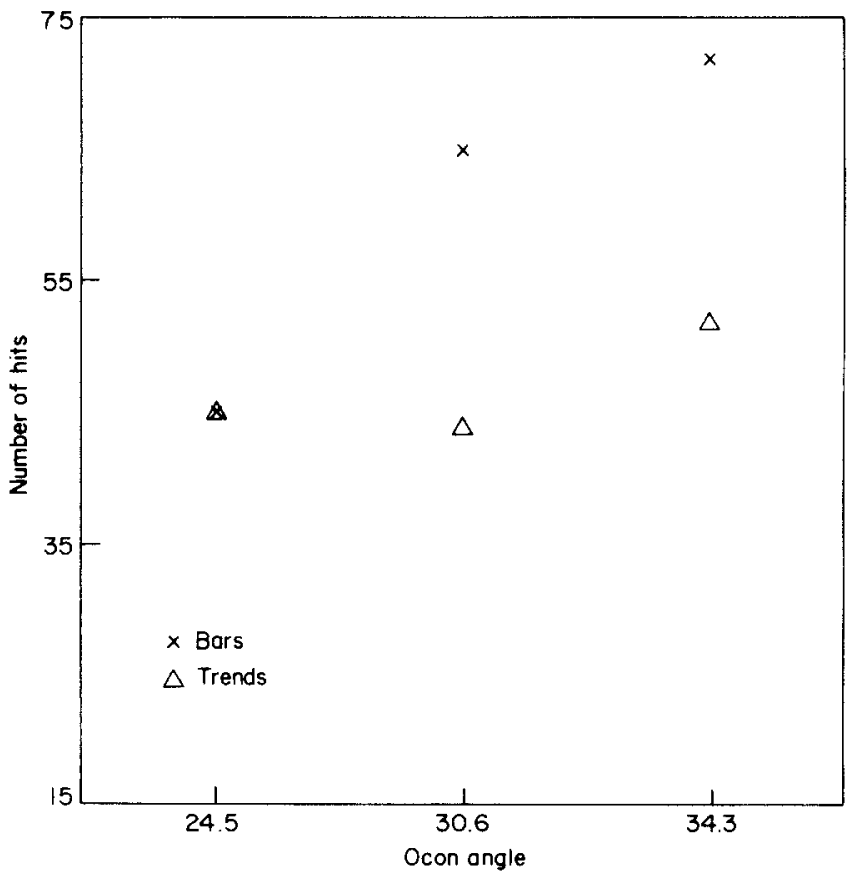

Fig. 4 Hit frequencies in the angle experiment

\section{Presentation mode}

The experimental data were subjected to the Wilcoxon matched-pairs signed rank test, except for the reaction times. Reaction times were tested for normality with the

Kolmogorov-Smirnov one-sample test. In all cases these times appeared to be distributed normally, and therefore they were analysed by an ANOVA. All data were tested for significance of the presentation mode effect. Table 2 shows the results for all four experiments. It can be seen that in all experiments significantly more hits were scored when bargraph presentation was used. In the frequency and angle experiments, the reaction time for bars is significantly longer than that for trends; however, in the amplitude experiment significantly longer RTs are associated with the trends. In all four experiments there is a significant interaction effect for RT, between presentation mode and the relevant blocks factor (filtering, ocon frequency, ocon amplitude and ocon angle respectively).

Only in the amplitude experiment is a significant difference present between the certainty scores (data not shown) for bars and trends, but only between those scores that are associated with hits. This means that subjective response certainty is not very sensitive to differences between presentation modes in actual performance.

The selection times associated with hits (data not shown) tend to be longer for trend pictures than for bars, although 


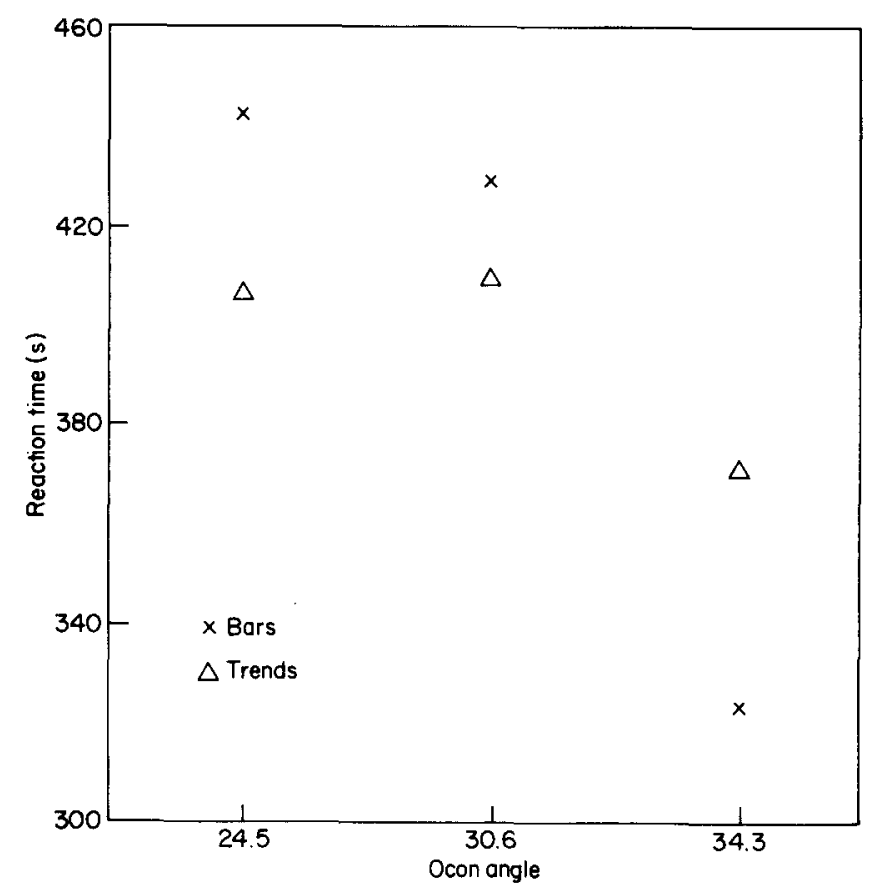

Fig. 5 Reaction times for trends and bars in the angle experiment

the difference is not significant in the frequency experiment. This result inay be due to the fact that trend pictures contain more (i e, historical) information than bargraph pictures, but differences in detection strategy may also have contributed to the selection time difference. Perhaps the longer build-up time for trend pictures stopped subjects from paging rapidly through the different pictures.

The longer keying times (data not shown) that are associated with trend presentation (only non-significant in the angle experiment) are a result of the longer and more complex identification code for trends. This latter fact may also have had some influence on the selection and reaction times.
False alarm codes produced by the subjects were tested for possible failures in remembering the correct code. This was achieved by comparing the false alarm against all present ocons for a match on all but one of the coding dimensions, the mismatch allowance for bargraph and unit number being restricted to one (e g, typing F3 instead of F2 or bar No 4 instead of 5) and accepting any colour mismatch. No 'close matches' of this kind were present.

\section{Separation of trends}

The general superiority of bargraphs with respect to the hit frequency raised a question regarding the cause of this phenomenon. The most obvious difference between bars and trends is in the absence and presence of historical information. If the mere presence of historical information degrades performance, combined trends should be associated with better performance than separated trends. Combined trends effectively contain much less historical information than their separated counterparts. Much of the information in combined trends is masked by other trends, because several trends share the same baseline.

Since the separation factor was implicitly included in the experiments, post hoc analysis of this factor could be performed.

The analysis was caried out for hit rates and hit-and-falsealarm frequencies. The results (Table 3) show that the performance with combined trends generally was better than with separated trends except in the amplitude experiment. In this latter experiment, combined trends were associated with a higher hit rate only in the condition with the largest amplitude (data not shown). In the other two conditions the hit rates were statistically identical.

The hit rates of the combined trends show a close correspondence with those of the bars. A test of the hit rates for bars against those for separated trends revealed significant differences in the filtering experiment (Wilcoxon's $z=3 \cdot 101$, $p<0.01)$, the frequency experiment $(z=2.353, p<0.025)$ and in the angle experiment $(z=2.722, p<0.01)$. In none of

Table 3: Performance data and results of Wilcoxon's matched pairs signed rank test of separated and combined trends

\begin{tabular}{llcccc}
\hline Experiment & Dependent variable & Separated & Combined & Wilcoxon's z & P-value \\
\hline Filtering & Hits (n) & 48 & 59 & 2.000 & 0.045 \\
& False alarms (n) & 74 & 45 & 2.045 & 0.041 \\
& Hit rate (\%) & 40 & 62 & 2.611 & 0.009 \\
\hline Frequency & Hits (n) & 29 & 63 & 3.059 & 0.002 \\
& False alarms (n) & 40 & 39 & 0.357 & 0.721 \\
& Hit rate (\%) & 38 & 68 & 2.756 & 0.006 \\
\hline Amplitude & Hits (n) & 65 & 76 & 1.490 & 0.136 \\
& False alarms (n) & 89 & 71 & 0.408 & 0.683 \\
& Hit rate (\%) & 57 & 61 & 0.909 & 0.363 \\
\hline Angle & Hits (n) & 61 & 80 & 2.012 & 0.044 \\
& False alarms (n) & 64 & 44 & 1.817 & 0.069 \\
& Hit rate (\%) & 57 & 77 & 3.413 & 0.001 \\
\hline
\end{tabular}


the experiments were hit rate differences present between bars and combined trends.

These results support the notion that the availability of historical information hampers the early detection of oncoming off-normals.

\section{Keying time}

The keying times for trends were generally longer than those for bars. The extra coding dimension necessary for the identification of trends (colour and record for trends $v s$ position only for bars) thus led to longer keying times, even though the required number of keystrokes was the same in both cases.

\section{Subjective data}

Answers to the questionnaire showed that some of the subjects favoured bars and some trends. Regardless of their preference, subjects performed better with bars than with (separated) trends. The evaluation of their own performance did not bear any relation to actual performance. Such a discrepancy between subjective assessments and objective measurements is a reason for some caution as regards our findings.

The certainty scores showed no clear pattern. A presentation mode effect was only present in the amplitude experiment. In this experiment the certainty scores for hits were higher for trends than for bars. In the filtering experiment, certainty increased with decreased filtering, but only in bar graph mode.

\section{Conclusions}

The different filterings applied to the signals did have effects on ocon detection performance. However, these effects are not clearcut and it is suggested that subjects may have adapted their detection and decision behaviour to changing demands. The angle of departure of ocons affected ocon detection performance, and especially reaction times. In all experiments the number of hits was higher for bar than for trend presentation (trend presentation consisting of both separated and combined trends). Better ocon detection performance was obtained with combined trends than with separated trends; performance with combined trends was similar to that with bars, while performance with separated trends was significantly worse than with bars.

The performance differences associated with the degree of filtering and ocon angle indicate that factors that contribute to differences between ocon and signal are determinants of ocon detection performance. The final size of deviations is less salient in this respect, at least in the range investigated.
These results have practical implications for the design of information displays in process control. The aim should be to optimise the perceptual separation between oncoming offnormals and signals containing no deviations. This may be achieved in various ways. The use of combined trends or bargraphs has been mentioned, but other solutions like preprocessing of data or scaling may be found.

A consistent and sizeable difference in ocon detection performance could be attributed to presentation mode. Differences in the build-up times for trends and bars may have had an effect on detection performance. There is no indication, however, that the longer build-up time for trends has led to longer reaction times: RT for trends is not longer than for bars, except in the amplitude experiment. Separated trends have been shown to produce inferior detection results. It is suggested that this difference must be traced to the inhibiting effect of the presence of historical information; further research is needed to investigate this in depth.

Experts in the field of process control tend to favour trend display over presentation of contemporary information only. The present research shows that, for some operator tasks, this may not invariably lead to optimal operator interface design solutions.

\section{References}

Dallimonti, R. 1980, Principles of design for man-machine interfaces in process control. In: Kompass, E.J., and Williams, T.J. (Eds), Man-machine interfaces for industrial control, Control Engineering.

Droffelaar, H. van. 1975, In: Schuh, P., and Sprague, P.A. (Eds), Productivity and man, RKW, Frankfurt.

Pousse, J.H. 1983, Structuring the process information on VDUs: first year report, Ergonomics Group, Twente University of Technology, Report WE 83/110/CT, Enschede.

Schaik, P. van. 1984, Structuring of process information on VDUs: experiments with trend/unit pictures, Ergonomics Group, Twente University of Technology, Report WE 84/119/WMW, Enschede.

Spenkelink, G.P.J. 1987, Structuring of process information on VDUs. Fourth year report I, Ergonomics Group, Twente University of Technology, Report WE 87/019/WMW, Enschede.

Weerdmeester, B.A. 1986, Structuring of process information on VDUs. Third year report, Ergonomics Group, Twente University of Technology, Report WE 86/112/CT, Enschede,

Zwaga, H.J.G., and Veldkamp, M. 1984, Evaluation of integrated control and supervision systems, Ergonomics problems in process operations, European Federation of Chemical Engineering, 133-146. 